the $\beta$ Pic system, and the timescale for the formation of planets in this and perhaps all planetary systems.

The outflowing gas detected by Bruhweiler et al., in observations of ionized iron (FE II) near a wavelength of $260 \mathrm{~nm}$, made with the International Ultraviolet Explorer (IUE) satellite, reaches a maximum velocity of only $60 \mathrm{~km} \mathrm{~s}^{-1}$, whereas the photospheric escape velocity for $\beta$ Pic is close to $700 \mathrm{~km} \mathrm{~s}^{-1}$. This gravitationally bound gas must eventually reverse course and become infalling gas. A gas component that experiences both mass ejection and infall is probably of stellar, rather than cometary origin. This is the first demonstra- tion that gas flows both inwards and outwards at $\beta$ Pic. Although both the rates of mass infall and outflow are variable, the largest measured infall event is $\mathbf{5 0}$ times more massive than the largest of the two observed outflow events. Although these observations demonstrate that some of the circumstellar plasma may be of stellar origin, they also show that stellar pulsations cannot account for all of the gas. Star-grazing comets are still the only viable explanation for producing much of the infalling gas.

David A. Weintraub is in the Department of Astronomy, University of Florida, Gainsville, Florida 32611, USA.

\title{
Real science at last
}

\section{David Lindley}

Astronomers working with the Hubble Space Telescope (HST) are no longer preoccupied with proving to the public that the flaws in the satellite's optics were not fatal. After six months of spectacular images derived from the telescope's testing runs, the first new scientific data are being gathered, and several unexpected observations were presented at a seminar* last month.

Much of the interest is in data obtained

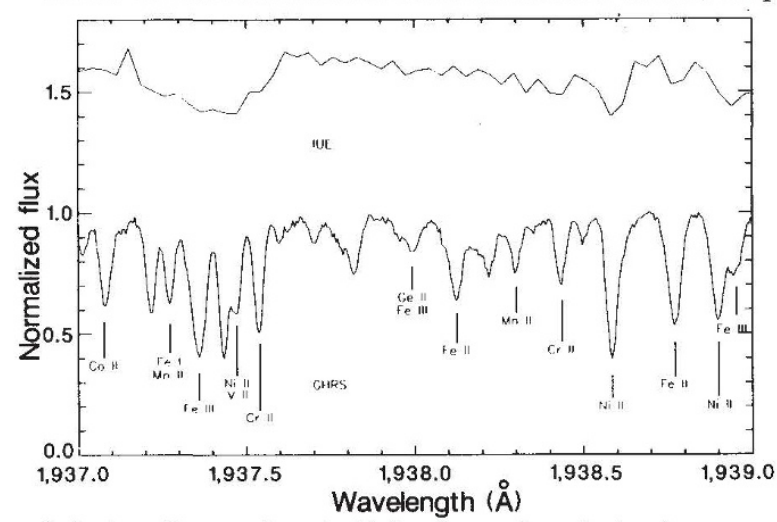

FIG 1. New (bottom) and old (top) spectra of $\chi$ Lupi. by the HST's two spectrometers, the highresolution and faint-objects spectrographs (HRS and FOS), both designed to operate primarily at ultraviolet wavelengths to which the Earth's atmosphere is opaque. The spectrum shown above (Fig. 1; D. Leckrone et al., Goddard Space Flight Center), a 2-ångstrom-wavelength interval of measurements of the hot, chemically peculiar star $\chi$ Lupi, shows what the HRS can achieve in comparison the best that had previously been obtained by the long-serving International Ultraviolet Explorer (IUE). The greatly magnified spectral resolution turns a wiggly line into a forest of atomic identifications. The advantage of ultraviolet stellar spectroscopy is that it includes low ionization lines of almost all the elements, and when the elements in question are of relatively low abundance, only the low ionization lines may be *Space Telescope Science institute, 17 May 1991 detectable at all.

Further spectra of $\chi$ Lupi turned up some novelties, and deepened some previous mysteries. Traces of ruthenium and arsenic were found, the first discovery of these elements in a non-solar astronomical spectrum. And the abundances of mercury and platinum, already known to be anomalously large, were estimated at $10^{5}$ and $10^{4}$ times solar abunestimated at $10^{5}$ and $10^{4}$ times solar abun-
dance, respectively. The puzzle is that $\chi$ Lupi has the brightness and colour of a relatively young, hot massive star, but has the heavy-element abundances that would be expected of a cool, highly evolved star after a lifetime of nucleosynthesis.

Spectroscopy also produced results of important but uncertain cosmological significance. For some years, observations from the ground have shown up dense patterns of absorption lines in the spectra of quasars, the most distant of astronomical objects; the lines have been attributed to the presence of hydrogen clouds at intermediate distances. The further away the clouds are, the more their Lyman- $\alpha$ absorption line is redshifted from the ultraviolet towards longer wavelengths in the optical waveband, by cosmological expansion. Optical observations have suggested that the density of Lyman- $\alpha$ clouds decreases to shorter wavelengths, meaning that closer and therefore older clouds are less common than more distant and therefore younger ones. This hinted that the clouds were some sort of leftover from galaxy formation.

But measurements from both HRS and FOS found over a dozen Lyman- $\alpha$ clouds in a section of the ultraviolet spectrum of the bright quasar $3 \mathrm{C} 273$ in which only one would have been predicted by extrapolation from previous results (Ray Weymann, Mount Wilson and Las Campanas Observa-

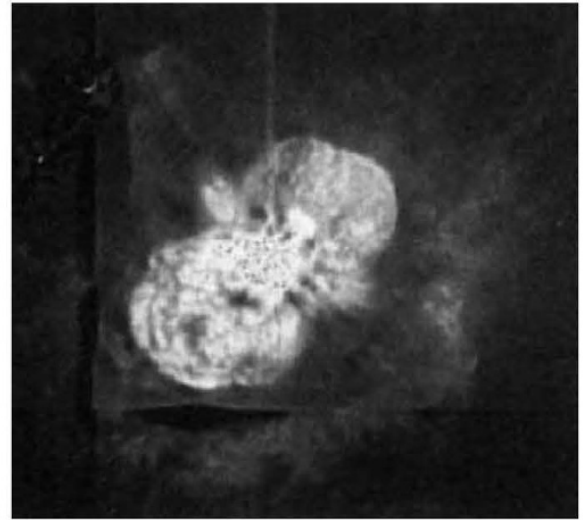

FIG 2. $\eta$ Carinae, as seen by the HST.

tories). Before HST, no instrument had had both the sensitivity and spectral resolution to search for Lyman- $\alpha$ clouds in the ultraviolet; a tentative identification of one absorption line by IUE turned into several lines in the HRS data.

The quantity of hydrogen implicated in a typical Lyman- $\alpha$ cloud is a column density of about $10^{14}$ atoms per $\mathrm{cm}^{2}$ - a nanogram, spread out over an unknown but presumably large diameter. Even clouds close by, identified by HST spectroscopy, will be impossible to detect in any other way, so that their cosmological origin and significance must be deduced from the most meagre of data.

These spectroscopic results contained most of the latest news, but no HST seminar would be complete without images. The bright star $\eta$ Carinae provides a most striking 'before and after' comparison. The star is of historical note: it flared from a nondescript 6 th magnitude to a remarkable -1 , only just less bright than Sirius, in the middle of the last century, then faded over the next decades back to 6 . The best guess at the structure, building on what was previously known of its history and on barely resolved images from ground-based telescopes, was that the star shed a lot of mass violently, creating two bright lobes of hot gas pushing out along what was presumably the polar axis, along with a few other scraps of debris.

The new HST observations (Fig. 2), however, turn this picture not upside down, but through 90 degrees. The WFPC (WideField/Planetary Camera) image shows the lobes but reveals seeming jet-like structure at right angles to them (J. Hester, California Institute of Technology). On one side, the jet seems to run into a shock, presumably when it collides with the interstellar medium, and behind it is a curious ladder of bright lines, possibly some sort of standing wave. On the other side, the jet seems to have encountered a higher density of material, and pushes out a sort of 'mushroom cap' of expanding material. Regardless of all these details, the interpretation now is that the jets signify the polar axis, and that the bright lobes represent an equatorial torus seen in cross-section.

David Lindley is Associate Editor of Nature. 